\title{
Ethical aspects of the changing landscape for spinal muscular atrophy management in Australia
}

\author{
Ainsley J Newson, Lisa Dive, Julie Cini, \\ Ellen Hurley, Michelle A Farrar
}

This article is the third in a series of articles on important topics in neurology.

\section{Background}

New methods of detecting and treating spinal muscular atrophy (SMA) are now available, and the Australian SMA landscape is rapidly changing. These various interventions can be increasingly complex to navigate for both healthcare professionals and at-risk families.

\section{Objective}

The aim of this article is to describe how recent developments in SMA testing and treatment give rise to ethical considerations.

\section{Discussion}

Ethical issues in SMA detection and treatment arise for both individual interventions and how they integrate. A patient-centred approach can help general practitioners to navigate these issues.
IN 2017, the Australian Federal Health Minister Greg Hunt MP announced two funding items that had been highly anticipated by those affected by spinal muscular atrophy (SMA). First, there would be support for a large national research trial of reproductive genetic carrier screening - Mackenzie's Mission. Second, there would be funding to allow access to a new drug treatment for SMA.

Mackenzie's Mission is now underway, providing up to 8500 Australian couples with information to facilitate informed reproductive choices. Nusinersen can now be used in clinical treatment in people with certain types of SMA. The advent of such new therapies has profoundly changed the SMA disease course from a lethal to a treatable chronic condition.

That funding to both treat and enable reproductive choice about SMA was announced in the same budget was striking, as carrier screening and drug treatment represent very different routes to mitigating the consequences of SMA. Consequently, approaches to reproductive, prenatal and newborn genetic screening for SMA are shifting. Options for intervention are increasingly complex to navigate.

In this article, the ethical aspects of detecting and treating SMA in Australia are considered. Ethical considerations include navigating clinical uncertainty, protecting reproductive choice, the value of life with a disability, how cost effectiveness should be construed, balancing benefits and harms in screening, and managing parental expectations regarding novel treatments. How interventions to prevent and treat SMA should be assessed in relation to each other are discussed, and a multifaceted approach - with patient-centred care at is core-is recommended.

\section{Spinal muscular atrophy}

SMA is an inherited neuromuscular disease historically associated with high morbidity and mortality. A pathogenic variant in both copies of the survival of motor neuron 1 (SMN1) gene causes SMA, while the number of copies of the separate $S M N 2$ gene are inversely related to (but do not precisely determine) severity. ${ }^{1-4}$ Some types of SMA are lethal.

Adults can access $S M N 1$ carrier screening by purchasing a commercial test or (until publicly funded screening is available) by participating in Mackenzie's Mission. Newborn screening for SMA is being rolled out nationally.

There are now three approved SMA therapies in Australia. ${ }^{4}$ Nusinersen and risdiplam improve both survival and function, especially if given pre-symptomatically. Yet treatment cannot fix degenerated motor nerves. Nusinersen is listed on the Pharmaceutical 
Benefits Scheme (PBS), including for pre-symptomatic children with 1-2 copies of SMN2, costing $\$ 110,000$ per treatment. ${ }^{5}$ Three intrathecal treatments are required every year after an initial loading period. Risdiplam is a once daily orally administered small molecule capped at $\$ 400,000$ per year, with PBS reimbursement for children aged between two months and 18 years who developed symptoms before the age of three years.

Onasemnogene abeparvovec is a one-dose gene therapy that also has best results if administered pre-symptomatically. The Therapeutic Goods Association permits use in infants aged $<9$ months who carry 1-3 copies of SMN2. Overseas this treatment costs $>\$ 2$ million; it is currently being considered for PBS listing. ${ }^{6}$

General practitioners (GPs) may encounter a range of clinical scenarios raising ethical issues. Some examples are detailed in Boxes 1-3.

\section{Preventing and treating SMA: Ethical considerations \\ Reproductive genetic carrier screening and prenatal diagnosis}

Reproductive genetic carrier screening (RCS) provides couples with information about their chance of having a child affected by severe recessive or X-linked genetic conditions. It can be undertaken prior to, or in the early stages of, pregnancy. SMN1 was one of three genes recommended for RCS funding by the Medicare Services Advisory Committee in $2020,{ }^{7}$ although no funding has yet been announced. SMN1 is currently being screened for in the Mackenzie's Mission project and appears on almost all commercial screening panels.

If a couple is found to have a high chance of having a child with SMA, they may access further interventions, if they choose. These include prenatal diagnosis (PND) to either prepare for, or avoid, the birth of a child with SMA, or preimplantation genetic testing (PGT) to select an embryo without two copies of the SMN1 pathogenic variant.

RCS offers couples the opportunity to make reproductive decisions in accordance with their values. It has broad (but not unconditional) support from families living with SMA, on the basis that it will both reduce suffering and raise awareness. ${ }^{8}$ It is also unarguably more cost effective to implement a universal offer of RCS than it would be to treat all cases of SMA after birth. ${ }^{9}$

However, RCS raises ethical issues. ${ }^{10}$ For SMA specifically, identifying carriers of a pathogenic variant in the SMN1 gene does not, on its own, predict the severity of SMA that a child may develop. A further consideration is the wider debate on the place of disability and difference in society. ${ }^{10}$ It has been argued that prenatal screening is unjustly discriminatory, particularly when there is variable presentation. ${ }^{11}$ Reasons include an implicit purpose of eradicating disability and diminishing diversity, thereby devaluing people living with genetic conditions such as SMA. In addition, when RCS is widely available and publicly funded, it may (even inadvertently) promote assumptions that screening is the responsible thing to do. ${ }^{12}$

GPs should facilitate opportunities for their patients to consider these issues in the context of their reproductive choices. This includes referral to appropriate RCS

\section{Box 1. Case study: Fatima and Aashif}

\section{Case}

Fatima and Aashif come to see you at your practice in the Australian Capital Territory. They have two children: Badi, aged three years, and Grace, aged four months. Grace has treated spinal muscular atrophy (SMA), which was detected via newborn screening. Grace has two copies of the survival of motor neuron 2 (SMN2) gene and was predicted to develop SMA type 1 without treatment. Consequently, she has received nusinersen since she was 28 days of age (and before symptom onset), with good outcomes so far. Fatima and Aashif have two concerns to discuss with you. First, they desire a third child and plan to start trying within the next 6-12 months. Both are opposed to termination of pregnancy but would consider prenatal testing in order to be prepared. Are they allowed to do this? What are the other options? Second, they are wondering about their older child, Badi. They are anxious to know whether he might be a carrier. Can they have him tested?

\section{Response}

Fatima and Aashif can access prenatal diagnosis, even if they do not plan to terminate. This can help them with planning. However, as Badi is clinically unaffected, Fatima and Aashif would be counselled that testing would usually be offered to Badi when he can make this decision for himself.

\section{Box 2. Case study: Martine}

\section{Case}

Martine books a long appointment. She arrives alone and brings a printout from a web page. Martine explains that she and her partner, Jeff, are planning a family and hope to become pregnant in the next six months. Martine read an article about carrier testing in a magazine and decided to get tested. She did an internet search and chose a company that tested for the most conditions at the lowest price. The carrier test was done via mail, using a provider based outside Australia. Martine had not realised until she chatted to a friend that some companies already offer this test in Australia, including some that offer pre- and post-test counselling. Martine is concerned that her test shows she carries a pathogenic variant in the survival of motor neuron 1 (SMN1) gene. She's not sure what this means, or what she can or should do next.

\section{Response}

Martine's situation illustrates the importance of ensuring that those accessing commercial carrier screening are doing so via appropriate providers. Several domestic testing companies provide reproductive genetic carrier screening with appropriate information and counselling. For conditions such as spinal muscular atrophy, it is vital that patients understand what their result means, how it is distinct from other tests such as those to detect chromosomal anomalies, and what options are available. 


\section{Box 3. Case study: Pablo}

\section{Case \\ Pablo was born a week ago in a large metropolitan hospital in NSW. Newborn screening (NBS) detected pathogenic variants in each copy of the survival of motor neuron 1 (SMN1) gene. After follow-up and confirmatory testing, Pablo is found to have three copies of the SMN2 gene, a genotype that has been detected in approximately $40 \%$ of babies who have mutations in each copy of SMN1 via NBS in NSW and the ACT. This means that Pablo will almost certainly develop spinal muscular atrophy (SMA) type 2 or 3 , but when and which type cannot be determined in advance. He is currently asymptomatic and so is not eligible to receive nusinersen with public funding. He may be able to obtain onasemnogene abeparvovec, but cost may be a barrier to access unless or until this treatment is listed on the Pharmaceutical Benefits Scheme (PBS). The choice at this time is to either watch and wait to then act quickly to commence nusinersen if symptoms develop, or to seek access to onasemnogene abeparvovec if funding can be identified. The risk with 'watch and wait' is that Pablo may have already had irreversible loss of motor function once clinical symptoms are evident. There is also a low chance that Pablo may be treated potentially unnecessarily with onasemnogene abeparvovec (if he is one of the rare number of children who have three copies of SMN2 yet do not develop clinical disease). Pablo's parents are not sure what to do.}

\section{Response}

While NBS has armed Pablo's family with the knowledge that he will almost certainly develop SMA at some point, currently there are barriers to accessing disease-modifying treatment. Pablo cannot access funded nusinersen until symptom onset. This 'watch and wait' approach may be stressful for his parents. He could also attempt to access onasemnogene abeparvovec, but this will require substantial funds. Such barriers to access are arguably an artefact of the rapid developments in SMA treatment and time lags associated with the complex Australian regulatory environment. The dilemma in this specific instance will hopefully be short lived, with onasemnogene abeparvovec progressing through assessment for PBS funding.

providers, such as those who offer the test together with counselling. Patients will need access to balanced information, including testimony regarding the lived experience of SMA and caring for those with this condition. This will help them to make decisions that are in line with their values. The Mackenzie's Mission project aims to investigate how RCS should be provided as a national program, including enabling equitable access.

\section{Newborn screening}

Newborn screening (NBS) is a proven public health initiative with high public trust. Because many patients with SMA experience a delay in diagnosis, NBS offers an important opportunity for early and equitable case identification. Many countries globally are now screening newborns for SMA, ${ }^{13,14}$ with NBS supported by the majority of SMA stakeholders. ${ }^{15-18}$ It has multiple benefits: enabling affected children to access care as early as possible and removing the necessity for parents to notice symptoms themselves. It also has negligible false positives and can inform parents' future reproductive planning. ${ }^{17,18}$

In Australia, SMA has recently been nationally recommended for NBS, based on evidence (including value for money) from a pilot SMA screening program in NSW and the ACT. ${ }^{17,19-21}$ As at January 2021, this pilot had detected 22 children from 252,081 babies screened. ${ }^{22}$

Nevertheless, NBS for SMA is not uncontroversial. ${ }^{3,13,17}$ If a person has more than three copy numbers of the SMN2 gene, there will be uncertainty regarding severity of presentation. There may be some newborns who are given a diagnosis that may not present until adulthood, leading to dilemmas about how and when to intervene. ${ }^{17,23}$ There may also be children identified via NBS for whom there is no funded treatment available. A defensible, transparent approach to determining 'screen positive' newborns will be required, such as treatment guidelines for infants who test positive. These concerns may be mitigated with long-term follow-up and, if indicated, adjustment of screening policy. ${ }^{13}$

NBS can also generate questions about other family members. Cascade testing from probands is currently offered to those who can decide about such testing for themselves. For immature minor siblings showing no clinical signs of SMA, testing is generally deferred until they can exercise their own choice. A further issue is that older siblings may not be able to access the same treatment, as there are specific PBS restrictions for each therapy based on age and symptoms. This scenario will require careful pre-test counselling and neurological assessment in partnership with paediatric multidisciplinary neuromuscular clinics and families.

\section{Therapeutic intervention}

While new therapies for SMA are extremely welcome, the rapid pace of their development is generating ethical complexity for health professionals and families. The long-term effects and outcomes of novel therapies for SMA remain uncertain. ${ }^{3,14,24-26}$ While they may prevent further decline, disease-modifying therapies are not a cure for SMA, and children who commence treatment after symptom onset might continue to live with impairment. ${ }^{27}$ It is important to manage parental optimism and hope, and ensure that benefits are not overestimated while possible harms are underplayed..$^{26,27}$

Equity concerns over access to treatment include restrictions related to age, treatment location, copies of SMN2 or disease progression. As noted, older siblings or adults in a family with a diagnosed newborn may not be able to access the same treatment. Families living rurally and remotely will incur travel costs and may experience interruptions to income in order to access clinical and procedural expertise. Presymptomatic children with $\geq 3$ copies of SMN2 cannot currently access reimbursed diseasemodifying therapies, and their families could struggle with the financial cost of accessing these, or with having to wait and undergo regular clinical observation to identify symptoms promptly.

Nusinersen and risdiplam are expensive drugs. Policy governing access to such 
medications must be transparent and address potential concerns such as value for money and fair pricing. ${ }^{28,29}$ As further longitudinal evidence about effectiveness emerges, criteria for treatment eligibility must be monitored, in collaboration with carers and SMA patients. A flexible, contextual approach is needed to balance the interests of all stakeholders. ${ }^{30}$

Onasemnogene abeparvovec is also costly, with public funding mechanisms actively being discussed. Families with affected children have previously sought treatment under a managed access program (allocated via a 'lottery' or purchased from Novartis). The latter has triggered crowdfunding: a practice that raises its own ethical issues, such as exacerbating inequality, the loss of privacy and directing resources away from those most likely to benefit. ${ }^{31}$ However, the upfront cost of this drug should be considered in relation to the fact that a single administration can potentially generate a lifetime of benefits. ${ }^{32}$

As more treatments for SMA and other rare diseases are developed, situations may emerge in which the best available treatment is not the funded treatment. Families might also require support to interpret evidence or to mitigate feelings of responsibility regarding accessing (or not accessing) the 'right' treatment for their child. They may also need support when deciding whether to initiate treatment. Present success rates and treatment burdens mean decisions to decline treatment are understandable. However, as therapies improve, a decision to decline treatment may become more contentious. Clinicians and families will need to work together to carefully balance projected gains to quality and length of life against the burden and cost of treatment. ${ }^{13,21}$

\section{(How) should SMA be prevented or treated?}

The close temporal development of primary and secondary interventions for SMA means ethical issues will also arise at their intersections. For example, what should a GP say to a couple who decline PND because SMA is 'treatable'? Should public funds be allocated to treating SMA when it is 'preventable'? Should NBS to detect SMA be funded once RCS is universally available?

These are complex questions, and fully addressing them is beyond the limits of this article. However, some overarching points can be made. First, it is important to consider the 'impairment experience' when making ethical assessments about SMA interventions. Interestingly, evidence suggests that those living with less severe forms of SMA are more likely to support RCS or NBS than those with more severe types. ${ }^{33}$ This may be because those with more severe types construe SMA as part of their inherent personal identity, whereas those with less severe types may have had a period of good health before symptom onset, and as such perceive SMA as something it is justifiable to avoid. ${ }^{34}$ Relatedly, people with no lived experience of SMA are likely to hold a more negative perception of this condition. ${ }^{13,35}$ Severity is also a complex concept. One practical way to engage with such experiences is to ensure those with a high chance of having a child with SMA are referred to support groups such as SMA Australia. This will help ensure that notions of severity are informed by both patient and clinical insights. ${ }^{34}$

Second, these contemporaneous developments mean that intervening in this condition is necessarily multifaceted. Rather than deciding that primary (RCS, PND) or secondary (NBS, treatment) interventions for SMA should be preferred, they should be seen as complementary. That is, healthcare for SMA is strengthened when all these interventions are available. GPs can engage their patients in discussion about the interventions that are relevant for them, supporting them to make decisions that reflect their considered values on aspects such as termination of pregnancy and raising a child who might have a disability.

Third, allocating funds in healthcare systems is complex. Allocating less funding to treating SMA will not necessarily mean more beds in intensive care units, for example. The varied interventions for SMA should not be prioritised against each other. RCS and PND are important interventions and can reduce family suffering. However, opting to have them should be a genuine choice for couples (based on factors such as respect for reproductive autonomy as well as considerations of equity and solidarity ${ }^{36}$ ), not one influenced by money to be saved in the healthcare system through not treating a child with SMA.

\section{Conclusion}

The current treatment landscape for SMA is rapidly changing, with new options for early testing and intervention that can dramatically change the disease outcome. These are summarised in this article, together with their associated ethical issues.

Patients will need support to sift through these options and issues. To work effectively with patients pre- and post-testing, general practices should ensure they have resources to access relevant information regarding the various screening and treatment options in SMA. Care should be supported by referral or consultation with expert specialists in 'grey' or difficult cases.

Patient-centred care that responds to each family's preferences and their specific context is crucial. The challenge of predicting the severity of SMA adds to this complexity, and there will not be one clear pathway to suit all patients. If patients understand their options, they can be supported to find the optimum approach to reflect their values and unique situation.

\section{Key points}

- SMA is a devastating inherited neurodegenerative condition. A gene test is available and can be used in preconception and prenatal testing, newborn screening and to inform clinical diagnosis.

- Detection and treatment of SMA is rapidly changing, with multiple interventions arising contemporaneously.

- Ethical issues in SMA detection and treatment include predicting severity, access to treatments and testing other family members.

- Ethical issues also arise as a result of the confluence of methods for detection and treatment. 


\section{- Involving existing SMA patients in discussions and providing patient- centred care are important.}

\section{Authors}

Ainsley J Newson BSc (Hons), LLB (Hons), PhD, Professor of Bioethics, Sydney Health Ethics, Sydney School of Public Health, Faculty of Medicine and Health, University of Sydney, NSW

Lisa Dive BA (Hons), PhD, MPhil (Med), Research Fellow, Sydney Health Ethics, Sydney School of Public Health, Faculty of Medicine and Health, University of Sydney, NSW

Julie Cini, Founder and CEO, Spinal Muscular Atrophy Australia, Hallam, Vic

Ellen Hurley MBBS, Neurology Fellow, Department of Neurology, Sydney Children's Hospital Network; Conjoint Associate Lecturer, School of Women's and Children's Health, UNSW Medicine, Sydney, NSW

Michelle A Farrar MBBS (Hons), FRACP, PhD, Associate Professor of Paediatric Neurology, School of Women's and Children's Health, UNSW Medicine, UNSW Sydney and Department of Neurology, Sydney Children's Hospital Network, Sydney, NSW

Competing interests: MAF reports personal fees from Biogen, Roche, Novartis, and grants from Biogen, all outside the submitted work.

Funding: AJN and LD received grant support from the Medical Research Future Fund: Australian Reproductive Genetic Carrier Screening Project (Mackenzie's Mission), GHFM73390 (MRFF-GMM). MAF received grant support from the National Health and Medical Research Council of Australia: Investigator grant (APP1194940).

Provenance and peer review: Commissioned, externally peer reviewed.

\section{Correspondence to:}

ainsley.newson@sydney.edu.au

\section{References}

1. Glascock J, Sampson J, Connolly AM, et al. Revised recommendations for the treatment of infants diagnosed with spinal muscular atrophy via newborn screening who have 4 copies of SMN2. J Neuromuscul Dis 2020;7(2):97-100 doi: 10.3233/JND-190468.

2. Farrar MA, Kiernan MC. Spinal muscular atrophy The dawning of a new era. Nat Rev Neurol 2020;16(11):593-94. doi: 10.1038/s41582-02000410-7.

3. Saffari A, Kölker S, Hoffmann GF, Weiler M, Ziegler A. Novel challenges in spinal muscular atrophy - How to screen and whom to treat? Ann Clin Transl Neurol 2018;6(1):197-205. doi: 10.1002/ acn3.689.

4. Davidson JE, Farrar MA. The changing therapeutic landscape of spinal muscular atrophy. Aust J Gen Pract 2022;51(1-2):38-42.

5. Australian Government Department of Health. The Pharmaceutical Benefits Scheme: Nusinersen. Canberra, ACT: DoH, 2020. Available at www.pbs. gov.au/medicine/item/11363C-11378W [Accessed 19 May 2021].

6. Australian Government Department of Health. Pharmaceutical Benefits Scheme medicine status website: Onasemnogene abeparvovec. Canberra, ACT: DoH, 2021. Available at www.pbs.gov.au/ medicinestatus/document/157.html [Accessed 19 May 2021].

7. Australian Government Department of Health Medical Services Advisory Committee. 1573 Reproductive carrier screening for fragile $X$ syndrome, spinal muscular atrophy and cystic fibrosis. Canberra, ACT: DoH, 2020. Available at www.msac.gov.au/internet/msac/publishing.nsf/ Content/1573-public [Accessed 19 May 2021].

8. Boardman FK, Young PJ, Griffiths FE. Population screening for spinal muscular atrophy: A mixed methods study of the views of affected families. Am J Med Genet A 2017:173(2):421-34. doi: 10.1002/ajmg.a.38031.

9. Gyngell C, Stark Z, Savulescu J. Drugs, genes and screens: The ethics of preventing and treating spinal muscular atrophy. Bioethics 2020;34(5):493-501. doi: 10.1111/bioe.12695.

10. Dive L, Newson AJ. Ethical issues in reproductive genetic carrier screening. Med J Aust 2021;214(4):165-67.e1. doi: 10.5694/mja2.50789.

11. Scully JL. Disability and the challenge of genomics. In: Gibson S, Prainsack B, Hilgartner S, Lamoreaux J, editors. Routledge handbook of genomics, health and society. London, UK: Routledge, 2018; p. 186-94.

12. van der Hout S, Dondorp W, de Wert G. The aims of expanded universal carrier screening: Autonomy, prevention, and responsible parenthood. Bioethics 2019;33(5):568-76. doi: 10.1111/bioe.12555.

13. Ross LF, Kwon JM. Spinal muscular atrophy: Past, present, and future. Neoreviews 2019;20(8):e437-51. doi: 10.1542/neo.20-8-e437.

14. Dangouloff T, Vrščaj E, Servais L, Osredkar D; SMA NBS World Study Group. Newborn screening programs for spinal muscular atrophy worldwide: Where we stand and where to go. Neuromuscul Disord 2021;31(6):574-82. doi: 10.1016/j.nmd.2021.03.007.

15. Boardman FK, Sadler C, Young PJ. Newborn genetic screening for spinal muscular atrophy in the UK: The views of the general population. Mol Genet Genomic Med 2018;6(1):99-108. doi: 10.1002/mgg3.353.

16. Boardman FK, Young PJ, Griffiths FE. Newborn screening for spinal muscular atrophy: The views of affected families and adults. Am J Med Genet A 2017;173(6):1546-61. doi: 10.1002/ajmg.a.38220.

17. Kariyawasam DST, D'Silva AM, Vetsch J, Wakefield CE, Wiley V, Farrar MA. 'We needed this': Perspectives of parents and healthcare professionals involved in a pilot newborn screening program for spinal muscular atrophy. EClinicalMedicine 2021;33:100742. doi: 10.1016/j. eclinm.2021.100742.

18. European Alliance for Newborn Screening in SMA. Spinal muscular atrophy: Screen at birth, save lives. Whitepaper version 1. Chipping Camden, UK: SMA Europe, 2021. Available at www.eamda.eu/ wp-content/uploads/2021/05/Spinal muscular atrophy Screen at birth save_lives_Whitepaper SMA_NBS_Alliance_v1_26March21.pdf [Accessed 17 May 2021].

19. Kariyawasam DST, Russell JS, Wiley V Alexander IE, Farrar MA. The implementation of newborn screening for spinal muscular atrophy: The Australian experience. Genet Med 2020;22(3):557-65. doi: 10.1038/s41436-0190673-0.

20. Australian Government Department of Health. Newborn bloodspot screening. Canberra, ACT: DoH, 2020. Available at www.health.gov.au/ health-topics/pregnancy-birth-and-baby/newbornbloodspot-screening [Accessed 10 June 2021].

21. Shih ST, Farrar MA, Wiley V, Chambers G. Newborn screening for spinal muscular atrophy with disease-modifying therapies: A costeffectiveness analysis. J Neurol Neurosurg Psychiatry 2021;92(12):1296-304. doi: 10.1136/ jnnp-2021-326344.
22. D'Silva AM, Kariyawasam DST, Best S, Wiley V, Farrar MA; NSW SMA NBS Study Group. Integrating newborn screening for spinal muscular atrophy into health care systems: an Australian pilot programme. Dev Med Child Neurol 2021. doi: 10.1111/dmcn.15117. Epub ahead of print.

23. Müller-Felber W, Vill K, Schwartz O, et al. Infants diagnosed with spinal muscular atrophy and 4 SMN2 copies through newborn screening Opportunity or burden? J Neuromuscul Dis 2020:7(2):109-17. doi: 10.3233/JND-200475.

24. Agosto C, Salamon E, Divisic A, et al. Do we always need to treat patients with spinal muscular atrophy? A personal view and experience. Orphanet J Rare Dis 2021;16(1):78. doi: 10.1186/ s13023-020-01593-4.

25. Audic F, de la Banda MGG, Bernoux D, et al. Effects of nusinersen after one year of treatment in 123 children with SMA type 1 or 2: A French real-life observational study. Orphanet J Rare Dis 2020:15(1):148. doi: 10.1186/s13023-020-01414-8.

26. Crawford TO, Sumner CJ. Assuring long-term safety of highly effective gene-modulating therapeutics for rare diseases. I Clin Invest 2021:131(15):e152817. doi: 10.1172/JCl152817.

27. King NMP, Bishop CE. New treatments for serious conditions: Ethical implications. Gene Ther 2017;24(9):534-38. doi: 10.1038/gt.2017.32.

28. Prasad V. Nusinersen for spinal muscular atrophy: Are we paying too much for too little? JAMA Pediatr 2018;172(2):123-25. doi: 10.1001/ jamapediatrics.2017.4360

29. Kerpel-Fronius S, Baroutsou V, Becker S, et al. Development and use of gene therapy orphan drugs - Ethical needs for a broader cooperation between the pharmaceutical industry and society. Front Med (Lausanne) 2020;7:608249. doi: 10.3389/fmed.2020.608249.

30. Benini F, Salamon E, Divisic A, Maghini Agosto C. Acknowledging limits: Statistics and the child's quality of life in spinal muscular atrophy. J Paediatr Child Health 2020;56(6):995-96. doi: 10.1111/jpc.14959.

31. Livingstone A, Servais L, Wilkinson DJC. Crowdfunding for neuromuscular disease treatment: The ethical implications. Lancet Neurol 2021:20(10):788-89. doi: 10.1016/S14744422(21)00266-0.

32. Garrison Jr LP, Jiao B, Dabbous O. Gene therapy may not be as expensive as people think: Challenges in assessing the value of single and short-term therapies. J Manag Care Spec Pharm 2021;27(5):674-81. doi: 10.18553/ jmcp.2021.27.5.674

33. Boardman FK, Young PJ, Griffiths FE. Impairment experiences, identity and attitudes towards genetic screening: The views of people with spinal muscular atrophy. J Genet Couns 2018;27(1):69-84. doi: 10.1007/s10897-017-0122-7.

34. Boardman FK, Clark CC. What is a 'serious' genetic condition? The perceptions of people living with genetic conditions. Eur J Hum Genet 2021. doi: 10.1038/s41431-021-00962-2. Epub ahead of print.

35. Boardman FK, Young PJ, Warren O, Griffiths FE. The role of experiential knowledge within attitudes towards genetic carrier screening: A comparison of people with and without experience of spinal muscular atrophy. Health Expect 2018;21(1):201-11. doi: 10.1111/hex.12602. Epub 2017 Jul 13.

36. Dive L, Newson AJ. Ethics of reproductive genetic carrier screening: From the clinic to the population. Public Health Ethics 2021;14(2):202-17. doi: 101093/phe/phab017. 